\title{
Advancing Models and Theories for Digital Behavior Change Interventions
}

Eric B. Hekler, PhD,${ }^{1}$ Susan Michie, DPhil, ${ }^{2}$ Daniel E. Rivera, $\mathrm{PhD},{ }^{3}$ Linda M. Collins, $\mathrm{PhD},{ }^{4}$

Misha Pavel, PhD,${ }^{5}$ Holly Jimison, PhD,${ }^{5}$ Claire Garnett, MSc, ${ }^{2}$ Skye Parral, MS, ${ }^{6}$ Donna

Spruijt-Metz, $\mathrm{PhD}^{6}$

From the ${ }^{1}$ School of Nutrition and Health Promotion, Arizona State University, Phoenix,

Arizona; ${ }^{2}$ Centre for Behaviour Change, University College London, London, United Kingdom;

${ }^{3}$ Fulton College of Engineering, Arizona State University, Tempe, Arizona; ${ }^{4}$ Methodology

Center, Pennsylvania State University, University Park, Pennsylvania; ${ }^{5}$ Consortium on

Technology for Proactive Care, Northeastern University, Boston, Massachusetts; and ${ }^{6}$ Center for

Economic and Social Research, University of Southern California, Los Angeles, California

Address correspondence to: Eric B. Hekler, PhD, School of Nutrition and Health Promotion, Arizona State University, 500 N. 3rd St., Phoenix AZ 85003. E-mail: ehekler@asu.edu. 
To be suitable for informing digital behavior change interventions, theories and models of behavior change need to capture individual variation and changes over time. The aim of this paper is to provide recommendations for development of models and theories that are informed by, and can inform, digital behavior change interventions based on discussions by international experts, including behavioral, computer, and health scientists and engineers. The proposed framework stipulates the use of a state-space representation to define when, where, for whom, and in what state for that person, an intervention will produce a targeted effect. The "state" is that of the individual based on multiple variables that define the "space" when a mechanism of action may produce the effect. A state-space representation can be used to help guide theorizing and identify crossdisciplinary methodologic strategies for improving measurement, experimental design, and analysis that can feasibly match the complexity of real-world behavior change via digital behavior change interventions. 


\section{Introduction}

A central task in science is the development and refinement of theories. A crossdisciplinary consensus definition of theory is "... a set of concepts and/or statements which specify how phenomena relate to each other. Theory provides an organizing description of a system that accounts for what is known, and explains and predicts phenomena." ${ }^{\prime 1}$ For health behavior change, theories provide a mechanism to encapsulate previous knowledge about how variations in causal factor(s) (e.g., an intervention) produce a desired effect (e.g., behavior change). Theory is useful because it provides explanations and predictions that support the generalization of findings from past work into future areas of inquiry and use. ${ }^{2,3}$

Theories of behavior change have been highly variable in the extent to which they achieve these goals. ${ }^{2}$ A review of behavior change theories with strict definitions of theory and behavior identified 83 theories. ${ }^{4,5}$ Of these, only three were judged to be comprehensive within their scope and there was generally poor specification, both in construct definitions and in the relationships between them. Further, most behavioral theories emphasized group-level and largely static generalization, meaning the theory supports explanations and predictions about average changes in outcomes in groups. ${ }^{6}$ Theory also has the potential to generate insights for specific individuals, particularly what might occur in the future for specific individuals. Ideally, a good theory will provide both group-level and individual-level generalizations. ${ }^{7-9}$

As described elsewhere, ${ }^{10}$ digital behavior change interventions (DBCIs) are interventions that employ digital technologies to encourage and support behavior change that will promote and maintain health, through primary or secondary prevention and management of health problems. 
Theories are key to effectively personalizing DBCIs. ${ }^{11}$ DBCIs facilitate health promotion by providing support in the "real world" to change specific behaviors in specific contexts and are used by individuals. ${ }^{12}$ They increasingly use information about a person to adapt provision of support to the unique and often changing needs of the individual. One class of DBCIs is the “just-in-time" adaptive intervention (JITAI). ${ }^{13}$ A JITAI provides support to individuals during just-in-time states when they have the opportunity to engage in a healthy behavior (or vulnerability to a negative behavior) and are receptive to support. ${ }^{14}$ JITAIs and DBCIs more generally require theories that take into account variations in individual characteristics and contexts and recognize that these variations will change over time. ${ }^{15}$ Current behavioral theories provide only limited insights for this type of intervention ${ }^{11}$ but are needed to manage the inherent complexity of real-world behavior change.

The aim of this paper is to provide recommendations for supporting the development of models and theories that are informed by, and can inform, DBCIs. The term "model" is used for a variety of purposes, but in general, models are sets of concepts, statements, or both that specify how constructs relate to each other to represent aspects of the world and can be precise and quantified or imprecise and qualitative. ${ }^{16}$ Theories are types of models that seek to explain phenomena that often invoke unobserved constructs to achieve this. ${ }^{16}$ Well-specified computational models, defined below, may be particularly useful for achieving the promise of highly personalized and precise DBCIs such as JITAIs. ${ }^{6}$ However, imprecisely specified models and theories can be useful. For example, a theory that stipulates that a construct such as "core identity" is an important driver of behavior can be useful in designing an intervention that seeks to change this in order, for example, to promote reduction in alcohol use. A great deal of work has already been 
done to advance strategies to use these more-imprecise models and theories for intervention development. ${ }^{17}$ Thus, the focus of this paper is on development of precise, quantifiable computational models as they are particularly relevant for DBCIs but also because the specification targets of computational models can support more careful theorizing even with imprecise models and theories.

Building on previous work, ${ }^{1,5,6,11,14,15,18-24}$ this paper:

1. specifies differences between broadly specified theories versus highly specified computational models that may be required for developing precise DBCIs;

2. states the case for more specific theorizing and testing on when, where, for whom, and in what state of the person a mechanism of action will produce an effect, ${ }^{23}$ by proposing the concept of "multidimensional generalization space," which specifies a set of dimensions along which contextual factors may vary to influence the size of effect of an intervention on an outcome (examples of such dimensions are aspects of target population and intervention setting; any given context can be specified as a point in that space); and

3. suggests crossdisciplinary methods to facilitate advancing the concept of multidimensional generalization space for DBCIs.

\section{Specification Requirements for Theories Versus Computational Models}

The differences between theories and computational models are related to the level of specification. Ideally, behavioral theories provide good specification of model structure and clear predictions about directionality and anticipated magnitudes of effects of a mechanism of action on an outcome. Model structure means clear specification of constructs and how constructs 
interact with one another, such as main effect relationships (i.e., self-efficacy is associated with behavior), moderation effects (i.e., the relationship between self-efficacy and behavior is moderated by self-regulatory skills), and mediation effects (i.e., the relationship between an intervention and behavior occurs via self-efficacy). ${ }^{25}$ These model structures are often visually described via path diagrams and are analyzed by techniques such as structural equation modeling. ${ }^{25-27}$ They may be tested by statistical estimation of effect sizes, which define the amount of variance statistically explained from an outcome variable by the predictor variable including specification of if there is a relationship, directionality, magnitude, and confidence in the relationship. For example, in one-meta-analysis, the mechanism of action of "teach to use prompts/cues," which is relevant to several theories, ${ }^{28}$ had an effect size estimate of $d=0.52$ for influencing physical activity. ${ }^{29}$

Within computational models, model structure and predictions about directionality and anticipated magnitudes of effects must be specified; thus, computational models can be conceptually seeded with well-validated theories. Computational models, however, require greater specification of the following two issues. The first is the dynamics of a relationship. This includes:

1. the anticipated timescale of an effect (i.e., amount of time when a meaningful change in a construct occurs, such as within seconds for heart rate and across years for the built environment) $)^{6,14}$

2. response patterns (i.e., the shape of a relationship, such as linear relationships or moredynamic step response options, such as feedback loops ${ }^{19}$ ); 
3. latency (i.e., the amount of time when one variable changes before observing change in the other); and

4. decay (i.e., the amount of time it takes for an effect to dissipate, as in operant learning theory $\left.{ }^{30,31}\right){ }^{6}$

For example, social cognitive theory predicts a reciprocal relationship between self-efficacy and behavior. ${ }^{25}$ As one goes up or down, the other goes up or down. Social cognitive theory does not provide clear specification on timescale (e.g., does self-efficacy change by the minute, hour, or day?), latency (e.g., does a change in self-efficacy immediately increase walking?), or decay (e.g., does the strength of the relationship between self-efficacy and walking diminish over time?), but these can be specified. ${ }^{25}$ The second issue to be specified is the multidimensional generalization space, which, again, specifies dimensions along which contextual factors may vary to influence the size of effect of an intervention on an outcome. Thus, a core difference is not only the specification on if there is a relationship, but also how that relationship functions over time and in context. ${ }^{19}$ Other work has provided careful discussion about the issues of dynamics $^{6,11,14,19,25}$ as an essential element of computational models.

Rothman ${ }^{23}$ and many others (e.g.. ${ }^{30}$ ) before have argued for the need for specification of when, where, and for whom a mechanism of action will produce a targeted effect through moderation testing. The argument is that behavioral theories, and by extension the development of theorydriven interventions, will become more precise if attention is placed on defining when, where, and for whom an intervention will and will not produce an effect. This argument is extended to the realm of DBCIs, which, as discussed already, are used in the real-world context where 
behaviors occur. As DBCIs are used in a real-world context, it implies the need for not only understanding when, where, and for whom an intervention will produce an effect but also having a clear understanding of the state of the person, thus implying the need for a state-space representation and the concept of a multidimensional generalization space.

\section{A State-Space Representation for Theorizing About Multidimensional}

\section{Generalization Space}

Multidimensional generalization spaces can be conceptualized using a state-space representation. Specifically, it is assumed that a participant's state can be represented in a multidimensional state space defined by variables that could feasibly impact the probability that an intervention will produce a desired effect. The point that represents an individual's current state is moving in accordance with the state-space transitions as predicted by different mathematical models such as a dynamical model of social cognitive theory. ${ }^{25}$

Given the instantaneous state of individuals, their responses can be characterized for any given intervention as the probability of the desired behavior. For simplicity, assume all other variables are constant but one (an unrealistic assumption but useful for demonstration). Based on that variable, differing probabilities are expected of the outcome occurring for two different interventions, A and B (Figure 1).

Theories of behavior change are not that simple and instead are based on the premise that individual, social, and environmental characteristics will change dynamically and interact to cause behavior change. For example, a cue to action to go for a walk (e.g., a text message saying, 
"Want to go for a walk?") could only inspire a walk if the state space of the person is appropriately receptive to this intervention. For example, Figure 2 is a plausible example of a multidimensional generalization space defined via three variables. The probability that a person goes for a walk increases if another person interested in walking is present (others present $=$ yes), if the person has a high overall opportunity to walk (e.g., $>5$ on a $0-10$ scale), and if they are not stressed (e.g., $<5$ on a $0-10$ scale).

Multidimensional generalization space is relevant for less time-intensive interventions. For example, a doctor-delivered motivational intervention to facilitate increased physical activity with a patient might only produce behavior change when the patient is sufficiently aware of the health risks of physical inactivity, is awake enough to engage in the interaction, and can fit in physical activity.

Theorizing about multidimensional generalization spaces for DBCIs is important for understanding concepts such as "teachable moments" ${ }^{31}$ and "just in time." ${ }^{14}$ A teachable moment, defined as events or circumstances that can lead a person to positive change, is widely referred to but has received little rigorous testing. ${ }^{31}$ DBCIs enable theoretic thinking and testing, for example when defining just-in-time states of opportunity and receptivity to an intervention. ${ }^{14}$ A person may have the opportunity to plan exercises for the week after dinner and right after putting their children to bed and be receptive to a small notification to do this planning from their smartphone when in that particular state. It is a plausible hypothesis that DBCIs will be more potent if they can be provided during these just-in-time states. Defining the multidimensional generalization space on when a mechanism of action will produce an effect will enable more 
rigorous testing of the teachable moment and just-in-time concepts, which has the potential to lead to more-precise and -potent DBCIs.

\section{Methodologic Strategies for Advancing Multidimensional Generalization}

\section{Space}

\section{Measurement}

A precondition of multidimensional generalization spaces for DBCIs is robust measurement strategies that can assess theoretic constructs in context, at the appropriate timescale, and with minimal burden to ensure continued data collection over time. Effective measurement of constructs is no small task, but is key, as it defines the level of precision that can be achieved within DBCIs. There are at least three areas that would advance measurement.

First, individuals that use digital technologies such as smartphones, computers, websites, and social media have a wide range of data gathered about them (e.g., all interactions a person has via e-mail). These data or "digital traces" are aggregated, connected, and organized and can be used for a variety of purposes such as highly targeted recommendations ${ }^{32}$ (e.g., "If you like this movie, then you will like this one"), or inferring psychological characteristics, such as

personality. ${ }^{33,34}$ If individuals gain access to their own digital traces, these data could be used to infer multidimensional generalization spaces. ${ }^{35}$ The use of digital traces can best be supported through strategies from computer science broadly labeled "machine learning." 36 The field of pervasive/ubiquitous computing, which studies the incorporation of computing capacity into everyday objects, provides insights from the "noise" of a digital trace, for example identifying meaningful patterns of breathing rates of individuals by translating small perturbations in the 
radio frequency signals sent and received from a WiFi hotspot (originally thought of as noise, small fluctuations in WiFi signal transmission appears to be influenced by the breathing of individuals in a systematic way). ${ }^{37}$

Second, there are important opportunities for developing ecologically valid sensors ${ }^{38,39}$ such as “wearables," which include fitness and stress tracking devices sensing and inferring target behaviors in context. ${ }^{38}$ These wearable technologies can enable increased measurement of realworld activities occurring in context, such as physical activity.

For constructs that cannot be measured directly (e.g., cognitions, perceptions), user-friendly strategies for measuring them in context are needed, with good progress being made in devising more- advanced ecological momentary assessment (EMA) techniques. ${ }^{40}$ For example, researchers are using "context-sensitive" EMA that utilizes sensors to infer the moments when it would be appropriate to ask for more-detailed questions. ${ }^{18,41}$ This type of work represents a logical path forward for EMA. These latent constructs are important to measure. For example, multidimensional generalization spaces should likely include the expected value of that action, which for an individual would include both the likelihood of the intended effect and the value (both cost and benefit) of the outcome.

As these measurement targets increasingly advance, they enable increased precision in the development of DBCIs that can be delivered efficiently when needed. Measurement alone cannot achieve this; advanced research methods and analytic strategies are also required. 


\section{Experimental Designs and Analytic Strategies}

Strategies inspired by both engineering and computer science can provide a logical empirical foundation for defining multidimensional generalization spaces for DBCIs. In engineering, methods from system identification ${ }^{42}$ present approaches to experimental design in behavioral intervention settings that are particularly useful for accomplishing the modeling of individual behavior and, by extension, can be supportive of multidimensional generalization spaces. System identification is an analytic technique that specifies the dynamic relationships between manipulated inputs (i.e., intervention components like goal-setting), disturbance variables (i.e., time-varying covariates that influence the outcome such as weather), endogenous state variables (i.e., time-varying covariates that influence the outcome but are aspects of the individual, such as stress), and outputs (i.e., behavioral outcomes such as steps) within a single-case, time-series context. The most common identification techniques apply strategies that build on the logic of regression in that they find solutions by minimizing squared errors. Methods from system identification are used extensively in practical engineering settings as a means for obtaining dynamic models that can be used in optimization strategies, such as model predictive control, to develop frameworks that support dynamic decision making, such as selection of a particular intervention option for a particular just-in-time state. ${ }^{43,44}$ Comprehensive system identification methodologies provide guidance regarding experimental design, model structure selection, parameter estimation for defining the dynamics, and validation of these idiographic models (e.g.,

a system identification experiment for physical activity ${ }^{45,46}$ ). This type of system identification experiment provides great opportunities for the empirical study of multidimensional generalization spaces. 
Inspired by computer science, experimental design and analytic approaches have been developed for a "micro-randomization" trial, which is also a useful experimental design for the study of multidimensional generalization spaces. ${ }^{20}$ The micro-randomization trial is a sequential factorial design that randomizes delivery/no delivery of an intervention at "decision points" when it is plausible that the intervention would be valuable. ${ }^{20}$ For example, every morning could be randomly assigned to delivering an intervention to help a person plan for that day. This approach supports empirically examining "time-varying moderation," which studies how factors that vary over time like context or stress can moderate the efficacy of an intervention. This can answer questions like: "Was the intervention only efficacious when a person was not stressed and at home?" This approach, which melds insights from computer science and statistics, provides appropriate data for examining multidimensional generalization spaces via time-varying moderation. $^{14}$

\section{Future Work}

There are four broad areas that require additional research attention. First, there should be increased movement toward theories and models that are as precise, quantitative, and testable as possible for describing the complexity of behavior change. Incremental advances toward precision can occur via specifying model structures, defining directionality and magnitude of relationships, dynamics, and multidimensional generalization spaces.

Second, the inherent complexity of behavior change implies that no one research group is likely to, alone, fully understand or model a phenomenon, particularly the multidimensional generalization spaces of an intervention, as this requires considerable resources. This points to 
the desirability of, and need for, collaborative research consortia. ${ }^{6}$ It also points to the need for the development of ontologies for understanding behavior as they provide a coherent structure for organizing and sharing insights across disparate research efforts. ${ }^{47}$ In brief, an ontology, as defined by the informatics tradition, is a highly structured description of terms/constructs and their inter-relationships. ${ }^{48}$ A key focus of ontologic work is to facilitate careful selection and definition of terms, such as behavior change techniques ${ }^{49}$ and mechanisms of action, and the proposed relationships between them. This type of work is essential to ensure scientists are studying the same concepts and thus will be critical for the study of multidimensional generalization spaces, as they will enable separate research efforts to be combined into more robust theories and computational models.

Third, is the importance of thinking of theories and computational models in integrated rather than siloed fashion, leading to collaboratively developed and evaluated theoretically based intervention modules. ${ }^{15}$ The study of human behavior involves careful understanding of under what conditions a mechanism of action will produce an effect. Behavioral theories are often treated as if they were generally true rather than specified well enough to define when they would and would not be useful for understanding a target phenomenon. ${ }^{23}$ It is essential for advancing behavioral science not only to focus on building computational models but also on the development of these models and behavioral theories in a collective mindset where each group of scientists are clearly specifying when a theory/model will and will not be useful. Theorizing about multidimensional generalization spaces is a logical target for supporting advancement in this area. 
Fourth, far greater work is required in the development of models that take into account and are predictive of changes over time that occur at an $\mathrm{N}=1$ or idiographic level. ${ }^{8,9}$ As discussed elsewhere, ${ }^{9}$ statistical analyses conducted within behavioral science tend to focus on an aggregation of data across individuals. For example, mixed model analyses ${ }^{50}$ parse variance to different "levels" such as distinguishing between-person and within-person variance explained for a target outcome. Between-person variance involves those factors that vary across individuals that are predictive of the outcome, such as differences in age, gender, or personality. Withinperson factors (which is a misnomer) focuses on how variations in predictor variables (e.g., daily variations in self-efficacy) on average across individuals are related to daily variations in an outcome measure of interest (e.g., daily variations in walking). ${ }^{51}$ In mixed model analyses, variations in factors that are specific to each individual (i.e., $\mathrm{N}=1$ ) are incorporated into the error terms and not the focus of modeling. ${ }^{50}$ These variations that are currently in the error term in mixed model analyses are the core focus of idiographic modeling.

The focus of idiographic modeling, such as system identification, ${ }^{52}$ attempts to generate highly specified models that describe how factors relate to one another for a specific individual to enable more robust prediction and eventual control (in the form of interventions) of the target phenomenon for a specific individual over time. This level of analysis is an essential target as it is a mechanism, along with well-specified multidimensional generalization spaces, for taking into account past data from an individual to foster more personalized predictions and decisions for that individual. Idiographic models are made possible, in part, with temporally dense time series data, which are increasingly available with DBCIs. ${ }^{22,27}$ Based on this, more careful modeling of $\mathrm{N}=1$ understanding of behavior ${ }^{8,9}$ is warranted and system identification is one 
logical approach as are computer science methods collectively labeled "reinforcement learning," such as the multi-arm bandit approach ${ }^{53}$ Further, these idiographic modeling techniques, as discussed earlier, are valuable for studying multidimensional generalization spaces thus further establishing their utility for enabling increasingly personalized and precise DBCIs.

\section{Conclusions and Next Steps}

In conclusion, DBCIs require theories and models of behavior change that capture and take into account individual variation and changes over time. There is a need for clear specification of facets of theories and models including model structure, directionality and magnitudes of effects, dynamics, and the multidimensional generalization space when a mechanism of action of a DBCI will produce a desired effect. Based on this work, there are at least three next steps. First, increased theorizing about dynamics and multidimensional generalization spaces is warranted to inform theories and models about behavior change and intervention effects. Although computational models can be useful for specifying this theorizing into quantifiable and falsifiable predictions, more general theorizing would be a valuable first step. Second, the concept of multidimensional generalization spaces is limited by the quality of measures of important constructs in context. Therefore, transdisciplinary research is needed to advance the understanding and measurement of these dynamic concepts and highlight particular opportunities in the realm of digital traces, wearable technologies, and EMA. Third, increased exploration and use of research methods and analytic techniques that can support more detailed study of both the dynamic relationships between constructs and the study of multidimensional generalization spaces is warranted. Uptake of these methods, such as system identification or the use of microrandomized trials, requires careful theorizing and thus can be supported via computational 
models. That said, progress can be made on the use of these methods even without fully specified computational models. ${ }^{14}$ These three steps can feasibly help to realize the vision of the DBCIs for improving public health and preventative care that is delineated in a sister piece in this special issue. $^{21}$ 


\section{Acknowledgments}

This 2016 theme issue of the American Journal of Preventive Medicine is supported by funding from the NIH Office of Behavioral and Social Sciences Research (OBSSR) to support the dissemination of research on digital health interventions, methods, and implications for preventive medicine.

This paper is one of the outputs of two workshops, one supported by the Medical Research Council (MRC)/National Institute for Health Research (NIHR) Methodology Research Programme (Principal Investigator [PI]: Susan Michie) and the Robert Wood Johnson Foundation (PI: Kevin Patrick), and the other by the National Science Foundation (PI: Donna Spruitj-Metz, proposal \#1539846).

The authors wish to thank the international experts that took part in these workshops and in a Dagstuhl seminar (15262) on lifelong behavior change technologies, which also informed this writing. Dr. Hekler and Dr. Rivera's work was provided, in part by a grant from the National Science Foundation (PI: Hekler, IIS-1449751). Dr. Hekler's work was supported, in part, by a grant from the Robert Wood Johnson Foundation (PI: Hekler, 71995). Drs. Jimison and Pavel's work was supported in part by the U.S. National Science Foundation under Grant 1407928, Tekes FiDiPro funding and by the National Institutes of Nursing Research Grant P20NR015320. Dr. Spruijt-Metz's effort was supported in part by the U.S. National Science Foundation under grant 1521740. 
Hekler, Spruijt-Metz, and Michie conceptualized, edited, and wrote several sections of the paper.

Rivera, Collins L., Pavel, Jimison, and Parral contributed substantively to the methods sections, Garnett contributed substantively to the sections on theory.

No financial disclosures were reported by the authors of this paper. 


\section{References}

1. Davis R, Campbell R, Hildon Z, Hobbs L, Michie S. Theories of behaviour and behaviour change across the social and behavioural sciences: a scoping review. Health Psychol Rev. 2015;9(3):323-344. http://dx.doi.org/10.1080/17437199.2014.941722.

2. Noar SM, Zimmerman RS. Health behavior theory and cumulative knowledge regarding health behaviors: are we moving in the right direction? Health Educ Res. 2005;20(3):275290. http://dx.doi.org/10.1093/her/cyg113.

3. Rothman AJ. "Is there nothing more practical than a good theory?": Why innovations and advances in health behavior change will arise if interventions are used to test and refine theory. Internat J Behav Nut Phys Act. 2004;1:11. http://dx.doi.org/10.1186/1479-5868$\underline{1-11}$.

4. Michie S, Campbell R, Brown J, West RR, Gainsforth H. ABC of Behaviour Change Theories: An essential resource for researchers, policy makers, and practitioners. London, UK: Silverback Publishing; 2014.

5. Prestwich A, Sniehotta FF, Whittington C, Dombrowski SU, Rogers L, Michie S. Does theory influence the effectiveness of health behavior interventions? Meta-analysis. Health Psychol. 2014;33(5):465. http://dx.doi.org/10.1037/a0032853.

6. Spruijt-Metz D, Hekler EB, Saranummi N, et al. Building new computational models to support health behavior change and maintenance: new opportunities in behavioral research. Translat Behav Med. 2015;5(3):335-346. http://dx.doi.org/10.1007/s13142-0150324-1. 
7. Shadish WR, Cook TD, Campbell DT. Experimental and quasi-experimental designs for generalized causal inference. Wadsworth Cengage Learning; 2002.

8. Molenaar P, Campbell C. The new person-specific paradigm in psychology. Curr Dir Psychol Sci. 2009;18(2):112-117. http://dx.doi.org/10.1111/j.1467-8721.2009.01619.x.

9. Molenaar PC. A manifesto on psychology as idiographic science: Bringing the person back into scientific psychology, this time forever. Measurement (Mahwah NJ). 2004;2(4):201-218. http://dx.doi.org/10.1207/s15366359mea0204_1.

10. Yardley L, Patrick K, Choudhury T, Michie S. Current issues and future directions for research into digital behavior change interventions. Am J Prev Med. 2016.

11. Riley WT, Rivera DE, Atienza AA, Nilsen W, Allison SM, Mermelstein R. Health behavior models in the age of mobile interventions: are our theories up to the task? Translat Behav Med. 2011;1(1):53-71. http://dx.doi.org/10.1007/s13142-011-0021-7.

12. Patrick K, Griswold WG, Raab F, Intille SS. Health and the mobile phone. Am J Prev Med. 2008;35(2):177-181. http://dx.doi.org/10.1016/j.amepre.2008.05.001.

13. Intille SS, Kukla C, Farzanfar R, Bakr W. Just-in-time technology to encourage incremental, dietary behavior change. In: AMIA Annual Symposium Proceedings; 2003: American Medical Informatics Association; 2003. p. 874.

14. Nahum-Shani I, Hekler EB, Spruijt-Metz D. Building health behavior models to guide the development of just-in-time adaptive interventions: A pragmatic framework. Health Psychol. 2016;34(Suppl):1209-1219. http://dx.doi.org/10.1037/hea0000306.

15. Hekler EB, Klasnja P, Riley WT, et al. Agile Science: Creating useful products for behavior change in the real-world. Translat Behav Med. Online February 26, 2016. http://dx.doi.org/10.1007/s13142-016-0395-7. 
16. Christmas S, Michie S, West R. Thinking about behaviour change: an interdisciplinary dialogue. London, UK: Silverback Publishing; 2016.

17. Michie S, Atkins L, West R. The behaviour change wheel: a guide to designing interventions. London, UK: Silverback Publishing; 2014.

18. Dunton GF, Atienza AA. The Need for Time-Intensive Information in Healthful Eating and Physical Activity Research: A Timely Topic. J Am Diet Assoc. 2009;109(1):30-35. http://dx.doi.org/10.1016/j.jada.2008.10.019.

19. Hekler EB, Buman MP, Poothakandiyl N, et al. Exploring behavioral markers of longterm physical activity maintenance: A case study of system identification modeling within a behavioral intervention. Health Educ Res. 2013;40(1S):51S-62S. http://dx.doi.org/10.1177/1090198113496787.

20. Klasnja P, Hekler EB, Shiffman S, et al. Micro-randomized trials: An experimental design for developing just-in-time adaptive interventions. Health Psychol. 2016;34(Suppl):1220-1228. http://dx.doi.org/10.1037/hea0000305.

21. Patrick K, Hekler EB, Estrin D, et al. Rapid rate of technological development and its implications for research on digital health behavior interventions. Am J Prev Med. 2016.

22. Rivera DE, Pew MD, Collins LM. Using engineering control principles to inform the design of adaptive interventions: A conceptual introduction. Drug Alcohol Depend. 2007;88(Suppl 2):S31-S40. http://dx.doi.org/10.1016/j.drugalcdep.2006.10.020.

23. Rothman AJ. Exploring connections between moderators and mediators: Commentary on subgroup analyses in intervention research. Prev Sci. 2013;14(2):189-192. http://dx.doi.org/10.1007/s11121-012-0333-y. 
24. Spruijt-Metz D, Nilsen W. Dynamic Models of Behavior for Just-in-Time Adaptive Interventions. IEEE Pervasive Comput. 2014(3):13-17. http://dx.doi.org/10.1109/MPRV.2014.46.

25. Riley WT, Martin CA, Rivera DE, et al. The Development of a Control Systems Model of Social Cognitive Theory. Translat Behav Med. Online November 9, 2016. http://dx.doi.org/10.1007/s13142-015-0356-6.

26. Anderson JC, Gerbing DW. Structural equation modeling in practice: A review and recommended two-step approach. Psychological Bulletin. 1988;103(3):411. http://dx.doi.org/10.1037/0033-2909.103.3.411.

27. Deshpande S, Rivera DE, Younger JW, Nandola NN. A control systems engineering approach for adaptive behavioral interventions: illustration with a fibromyalgia intervention. Translat Behav Med. 2014;4(3):275-289, Errratum in 4(3), p439.

28. Bandura A. Social Foundations of Thought and Action: A Social Cognitive Theory. Englewood Cliffs, NJ: Prentice Hall; 1986.

29. Olander EK, Fletcher H, Williams S, Atkinson L, Turner A, French DP. What are the most effective techniques in changing obese individuals' physical activity self-efficacy and behaviour: a systematic review and meta-analysis. Int J Behav Nutr Phys Act 2013;10(29):1-15. http://dx.doi.org/10.1186/1479-5868-10-29.

30. Van Bavel JJ, Mende-Siedlecki P, Brady WJ, Reinero DA. Contextual sensitivity in scientific reproducibility. Proc Natl Acad Sci U S A. 2016;113(23):6454-6459.

31. Lawson PJ, Flocke SA. Teachable moments for health behavior change: a concept analysis. Patient Educ Couns. 2009;76(1):25-30. http://dx.doi.org/10.1016/j.pec.2008.11.002. 
32. Resnick P, Varian HR. Recommender systems. Commun ACM. 1997;40(3):56-58. http://dx.doi.org/10.1145/245108.245121.

33. Golbeck J, Robles C, Turner K. Predicting personality with social media. In: $C H I^{\prime} 11$ extended abstracts on human factors in computing systems. ACM;2011:253-262. http://dx.doi.org/10.1145/1979742.1979614.

34. Zhou MX, Nichols J, Dignan T, Lohr S, Golbeck J, Pennebaker JW. Opportunities and risks of discovering personality traits from social media. In: Proceedings of the extended abstracts of the 32nd annual ACM conference on Human factors in computing systems (CHI'14). ACM; 2014:1081-1086. http://dx.doi.org/10.1145/2559206.2579408.

35. Estrin D. Small data, where n = me. Commun ACM. 2014;57(4):32-34. http://dx.doi.org/10.1145/2580944.

36. Witten IH, Frank E. Data Mining: Practical machine learning tools and techniques. Morgan Kaufmann; 2005.

37. Ravichandran R, Saba E, Chen K-Y, Goel M, Gupta S, Patel SN. WiBreathe: Estimating Respiration Rate Using Wireless Signals in Natural Settings in the Home. In: IEEE Pervasive Computing and Communications (PerCom), 2015 IEEE International Conference on. http://dx.doi.org/10.1109/PERCOM.2015.7146519.

38. Kumar S, Nilsen W, Pavel M, Srivastava M. Mobile health: Revolutionizing healthcare through trans-disciplinary research. Computer (Long Beach Calif). 2013;46(1):28-35.

39. Kumar S, Nilsen WJ, Abernethy A, et al. Mobile health technology evaluation: the mHealth evidence workshop. Am J Prev Med. 2013;45(2):228-236. http://dx.doi.org/10.1016/j.amepre.2013.03.017. 
40. Shiffman S, Stone AA, Hufford MR. Ecological momentary assessment. Ann Rev Clin Psychol. 2008;4:1-32. http://dx.doi.org/10.1146/annurev.clinpsy.3.022806.091415.

41. Dunton GF, Dzubur E, Kawabata K, Yanez B, Bo B, Intille S. Development of a smartphone application to measure physical activity using sensor-assisted self-report. Front Public Health. 2014;2:12. http://dx.doi.org/10.3389/fpubh.2014.00012.

42. Ljung L. System Identification: Theory for the user. PTR Prentice Hall Information and System Sciences Series; 1987;198.

43. Nandola NN, Rivera DE. An improved formulation of hybrid model predictive control with application to production-inventory systems. IEEE Trans Control Syst Technol. 2013;1:121-135. http://dx.doi.org/10.1109/TCST.2011.2177525.

44. Dong Y, Rivera DE, Downs DS, Savage JS, Thomas DM, Collins LM. Hybrid model predictive control for optimizing gestational weight gain behavioral interventions. Proc Am Control Conf. 2013:1970-1975.

45. Martin CA, Desphande S, Hekler EB, Rivera DE. A system identification approach for improving behavioral interventions based on social cognitive theory. 2015 American Control Conference (ACC). 2015; Chicago, IL:5878-5883. http://dx.doi.org/10.1109/ACC.2015.7172261.

46. Martin CA, Rivera DE, Riley WT, et al. A Dynamical Systems Model of Social Cognitive Theory. 2014 American Control Conference. 2014; Portland, OR:2407-2412. http://dx.doi.org/10.1109/ACC.2014.6859463.

47. Larsen K, Michie S, Hekler EB, et al. Behavior change interventions: The potential of ontologies for advancing science and practice. J Behav Med. In press. 
48. Arp R, Smith B, Spear AD. Building ontologies with basic formal ontology. Cambridge, MA: The MIT Press; 2015. http://dx.doi.org/10.7551/mitpress/9780262527811.001.0001.

49. Michie S, Wood C, Johnston M, Abraham C, Francis J, Hardeman W. Behaviour change techniques: the development and evaluation of a taxonomic method for reporting and describing behaviour change interventions. Health Technol Assess. 2015;19(99):1-188. http://dx.doi.org/10.3310/hta19990.

50. Singer JD, Willett JB. Applied Longitudinal Data Analysis: Modeling Change and Event Occurrence. New York: Oxford University Press; 2003. http://dx.doi.org/10.1093/acprof:oso/9780195152968.001.0001.

51. Hekler EB, Buman MP, Ahn D, Dunton GF, Atienza AA, King AC. Are daily fluctuations in perceived environment associated with walking? Psychol Health. 2012;27(9):1009-1020. http://dx.doi.org/10.1080/08870446.2011.645213.

52. Ljung L. System Identification: Theory for the use. 2nd Edition. Upper Saddle River, NJ: Prentice Hall; 1999. http://dx.doi.org/10.1002/047134608x.w1046.

53. Sutton RS, Barto AG. Reinforcement learning: An introduction. Boston, MA: MIT press; 1998. 


\section{List of Figures}

Figure 1. One variable visualization of a multidimensional generalization space.

Figure 2. Three-variable visualization of a multidimensional generalization space.

Note: The darker the shade, the increased likelihood that an intervention will produce the desired effect.

Figure 3. Take home messages. 
Table 1. Theories Versus Computational Models

\begin{tabular}{lll}
\hline Facets specified & Theory & Computational models \\
\hline & Model structure & Model structure \\
\hline & $\begin{array}{l}\text { Predicted directionality \& } \\
\text { magnitude of effects }\end{array}$ & $\begin{array}{l}\text { Predicted directionality and } \\
\text { magnitude of effects }\end{array}$ \\
\hline Advantages & Dynamics \\
& $\begin{array}{l}\text { Multidimensional } \\
\text { generalization space }\end{array}$ \\
& $\begin{array}{l}\text { Provides a conceptual } \\
\text { framework to organize } \\
\text { research efforts }\end{array}$ & $\begin{array}{l}\text { falsify complex predictions } \\
\text { related to dynamics and } \\
\text { multidimensional } \\
\text { generalization spaces }\end{array}$ \\
& & $\begin{array}{l}\text { Enables the use of simulation } \\
\text { to further study behavioral } \\
\text { phenomena }\end{array}$ \\
\hline
\end{tabular}




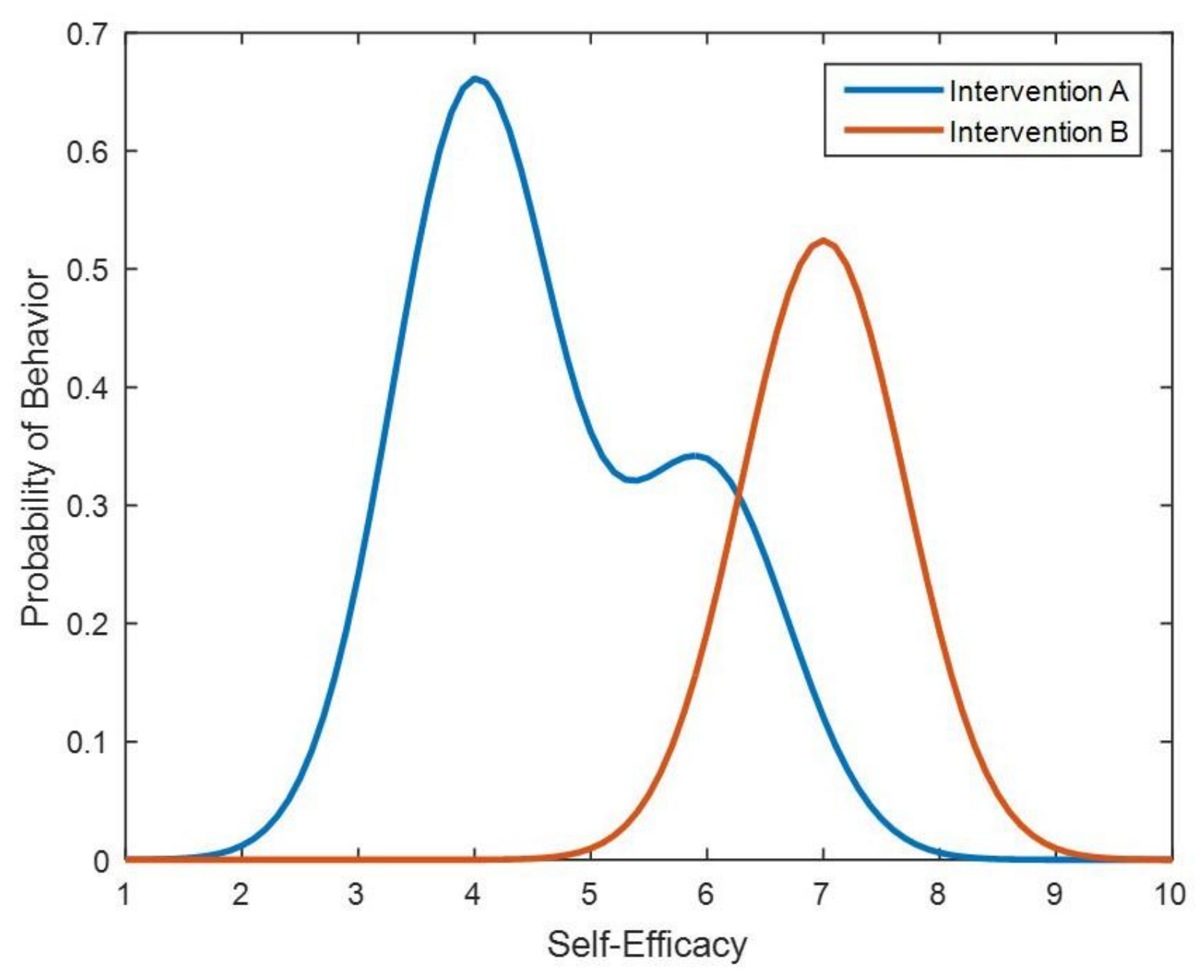




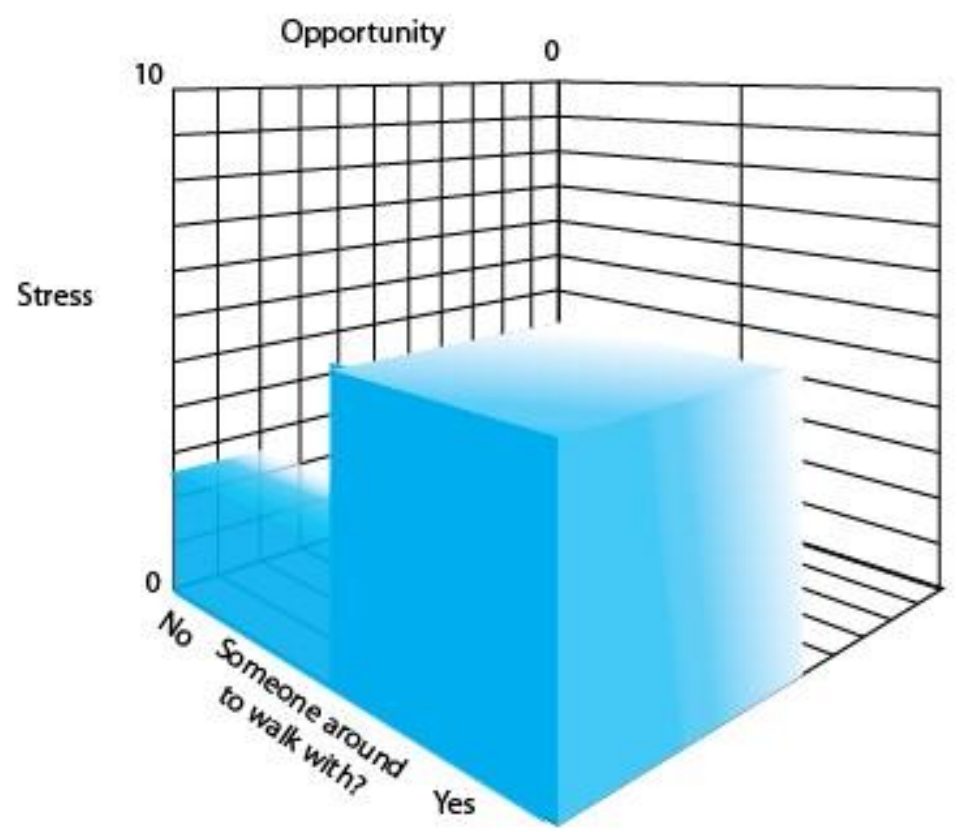


- Increased theorizing about dynamics and multidimensional generalization space - defined as specification of a set of dimensions along which contextual factors may vary to influence the size of effect of an intervention on an outcome (examples of such dimensions are aspects of target population and intervention setting; any given context can be specified as a point in that space) - is needed to support development and refinement of digital behavior change interventions.

- This theorizing can be supported via:

- increased use of computational models as a complement to more general theory development and refinement;

- a transdisciplinary research agenda to improve measurement of dynamics and multidimensional generalization spaces;

- increased use of research methods and analytic techniques that enable testing of dynamics and multidimensional generalization spaces.

- This could support more open scientific processes for collective theory development and refinement for digital health behavior change interventions. 